\section{Psoriasis: Spinaliome unter TNF-a-Hemmern}

\section{Eine TNF-a-Inhibition scheint das Risiko für ein Plattenepithelzellkarzinom um rund $80 \%$ zu erhöhen. Darauf deuten US-Versicherungsdaten.}

mmer wieder gibt es Befürchtungen, Biologika könnten das Krebsrisiko steigern. Hautärzte aus den USA haben sich nun gezielt die Krebsrate bei Psoriasispatienten unter einer systemischen Biologikabehandlung angeschaut. Sie konnten für ihre Analyse auf Angaben eines USVersicherers zurückgreifen. Berücksichtigt wurden knapp 5.900 Psoriasiskranke, die zwischen 1998 und 2011 eine systemische Behandlung bekommen hatten. Die Ärzte unterschieden dabei zwischen einer Therapie mit Biologika (2.285 Patienten), meist war dies ein TNF- $\alpha$-Hemmer, und einer Behandlung mit anderen Medikamenten, etwa Methotrexat, Retinoiden oder Cyclosporin (3.604 Patienten).

Patienten mit Biologika waren im Schnitt etwas jünger als solche ohne, schwerer krank, hatten häufiger eine Psoriasis-Arthritis und waren öfter mit UV-Licht behandelt worden. Überraschenderweise erkrankten die Patienten mit Biologika seltener an Tumoren als die ohne (8,2\% versus $11,5 \%)$. Wurden jedoch Alter, Geschlecht, Vortherapien und Komorbiditäten berücksichtigt, ergaben sich keine signifikanten Unterschiede zwischen den beiden Gruppen.

Nicht melanozytäre Hauttumoren traten mit $12,0 \%$ versus $11,4 \%$ bei den Biologikapatienten etwas häufiger auf. Wurden wiederum Alter und andere Unterschiede berücksichtigt, ergab sich sogar ein um $42 \%$ erhöhtes Risiko für solche Tumoren unter Biologika. Dieser Unterschied ließ sich im Wesentlichen auf eine erhöhte Rate von Plattenepithelzell-

karzinomen zurückführen. Daran erkrankten 4,8\% der Patienten mit Biologika, aber nur 3,7\% ohne. Nach diversen Adjustierungen konnten die Forscher daraus ein um $81 \%$ höheres Spinaliomrisiko unter Biologika ableiten.

Das Basaliomrisiko scheint mit TNFa-Hemmern ebenfalls höher zu sein, jedoch nur mit einem nicht signifikanten Unterschied von $23 \%$. In ähnlicher Weise zeigte sich ein um $57 \%$ erhöhtes Melanomrisiko - allerdings traten Melanome so selten auf, dass dieser Unterschied statistisch betrachtet nicht auffällig war.

Fazit: Die Tumorgefahr für Psoriasispatienten steigt insgesamt unter einer systemischen TNF- $\alpha$-Hemmer-Therapie nicht. Allerdings ergibt sich aus dieser Analyse ein deutlich erhöhtes Risiko für Spinaliome.

Thomas Müller

Asgari M et al. Malignancy rates in a large cohort of patients with systemically treated psoriasis in a managed care population.

J Am Acad Dermatol 2017; 76: 632-8

\title{
Melanom: Lymphknotendissektion auch therapeutisch von Nutzen?
}

\section{Eine Sentinellymphknotendissektion bei Melanompatienten könnte nicht nur einen diagnostischen, sondern auch einen therapeutischen Nutzen haben. Hinweise dafür liefert eine Studie mit fast 600 Patienten ohne Fernmetastasen.}

D a weiterhin Uneinigkeit darüber herrscht, ob das Entfernen von Mikrometastasen während der Sentinellymphknotendissektion (SLND) das Überleben beeinflusst, haben deutsche Dermatologen die SLND-Ergebnisse von 596 Melanompatienten, die zwischen 1996 und 2003 behandelt worden waren, mit denen von Patienten mit weiter lokaler Exzision verglichen.

Die Patienten hatten lokale Melanome der Haut mit einer Breslow-Dicke von mindestens $1 \mathrm{~mm}$. Die Primärtumoren wurden vier Wochen vor oder gleichzeitig mit der SLND exzidiert. Der Sicherheitsabstand lag zwischen 1 und $2 \mathrm{~cm}$. Nach 1996 wurden alle Patienten mit primärem Melanom und einer Breslow-Dicke von mindestens $1,5 \mathrm{~mm}$ sowie Patienten mit positiven Lymphknoten nach einer kompletten Lymphknotendissektion und fehlenden Fernmetastasen mit Interferon $\alpha$ behandelt. Seitdem unterlag das Therapieprotokoll Veränderungen.

Das mediane Follow-up lag in der SLND-Gruppe bei 73 Monaten, in der Vergleichsgruppe bei 91 Monaten. In Drei- bis Sechsmonatsintervallen wurden die Patienten unter anderem klinisch untersucht und die Lymphknoten beurteilt. Die mediane Tumordicke lag in der SLND-Gruppe bei $1,7 \mathrm{~mm}$, in der Vergleichsgruppe bei $1,8 \mathrm{~mm}$. Die meisten Tumoren wurden in der SLNDGruppe als Clark Level IV (60,7\%) klassifiziert. In der Vergleichsgruppe lag der Anteil mit 65,4\% ähnlich hoch.

Wie aus den Daten hervorgeht, lebten Patienten mit Dissektion des Wächterlymphknotens länger. Das tumorspezifi- sche Überleben lag in dieser Gruppe bei 102,7 Monaten, die Zehnjahresüberlebensrate betrug $74,9 \%$. In der Vergleichsgruppe waren es 97 Monate und 66,9\% Überlebensrate $(p=0,024)$. Noch deutlicher war der Unterschied zwischen den beiden Gruppen bei der durchschnittlichen Dauer bis zur Lymphknotenprogression. Sie betrug in der SLND-Gruppe 111 Monate, in der Vergleichsgruppe dagegen 98,5 Monate ( $<<0,001)$. Zur Krankheitsprogression kam es bei 20,8\% der Patienten in der SLND-Gruppe und bei $30,5 \%$ in der Vergleichsgruppe. Deutlicher war der Unterschied beim Anteil der Patienten mit Lymphknotenprogression: $8,2 \%$ versus $20,5 \%$.

Fazit: Melanompatienten scheinen von einer SLND zu profitieren. Einschränkend weisen die Autoren darauf hin, dass unklar bleibt, wie sich die SLND auf Patienten mit dünneren Melanomen auswirkt.

Peter Leiner

Geimer T et al. The impact of sentinel node dissection on disease-free and overall tumourspecific survival in melanoma patients: a single centre group-matched analysis of 1192 patients. J Eur Acad Dermatol Venereol 2017; 31: 629-35 